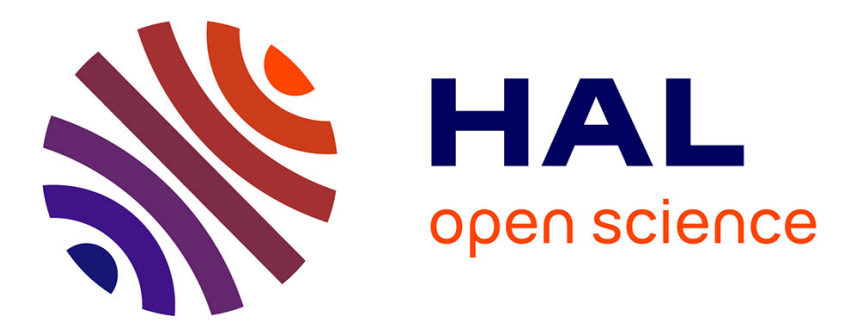

\title{
Complexity Results in Optimistic/Pessimistic Preference Reasoning
}

Christian Bessiere, Remi Coletta, Gaelle Hisler, Anastasia Paparrizou

\section{To cite this version:}

Christian Bessiere, Remi Coletta, Gaelle Hisler, Anastasia Paparrizou. Complexity Results in Optimistic/Pessimistic Preference Reasoning. ICTAI: International Conference on Tools with Artificial Intelligence, Nov 2016, San Jose, CA, United States. pp.930-937, 10.1109/ICTAI.2016.0144 . lirmm01987872

\section{HAL Id: lirmm-01987872 \\ https://hal-lirmm.ccsd.cnrs.fr/lirmm-01987872}

Submitted on 21 Jan 2019

HAL is a multi-disciplinary open access archive for the deposit and dissemination of scientific research documents, whether they are published or not. The documents may come from teaching and research institutions in France or abroad, or from public or private research centers.
L'archive ouverte pluridisciplinaire HAL, est destinée au dépôt et à la diffusion de documents scientifiques de niveau recherche, publiés ou non, émanant des établissements d'enseignement et de recherche français ou étrangers, des laboratoires publics ou privés. 


\title{
Complexity Results in Optimistic/Pessimistic Preference Reasoning
}

\author{
Christian Bessiere*, Remi Coletta* ${ }^{*}$, Gaelle Hisler*† and Anastasia Paparrizou* \\ * CNRS, University of Montpellier, France \\ $\dagger$ Tellmeplus, Montpellier, France \\ \{bessiere,coletta,hisler,paparrizou\}@lirmm.fr
}

\begin{abstract}
Preference reasoning is a central problem in decision support. There exist various ways to interpret a set of qualitative preferences. Conditional preference logics allow to deal with semantics such as optimistic, pessimistic, strong or not. In this paper, we study the complexity of the main problems in optimistic/pessimistic preference logic: undominated, consistency and dominance. We show that they are all NP-hard in general, with some becoming polynomial under specific semantics. Our second contribution is to show that the dominance problem, which has an online component in its definition, is compilable to polynomial time.
\end{abstract}

\section{INTRODUCTION}

Preferences appear in our everyday life each time we make a choice. When the number of alternatives becomes large, people/users unconsciously express preferences over them. Preferences help in making a faster decision rather than comparing an (potentially) exponential number of alternatives. Preference reasoning aims at supporting the user in making a choice reflecting her preferences. Reasoning with preferences is a topic of increasing interest in decision support.

Given a set of preferences defined by the user, determining the best choice(s) is one of the main problems in preference reasoning, called the undominated problem. Other main problems are the consistency of a set of preferences, or the ranking of pairs of outcomes, i.e. the dominance problem.

There exist two main ways to express preferences in the literature: one for quantitative preferences and one for qualitative preferences. The most popular formalism of quantitative preferences is the GAI-net (Generalized Additive Independence) [1]. GAI-net offers the advantage that dominance testing simply requires to compute the utility of each outcome. But, all quantitative preference formalisms suffer from the same drawback: how to specify the weights of the alternatives? It can be difficult for a user to state she prefers to buy a house with weight 0.7 rather than an apartment with weight 0.3 .

On the other hand, qualitative preferences are easier to be expressed by the user. CP-nets [2] are a well-known formalism of qualitative preference reasoning. CP-nets use a semantics called ceteris paribus (i.e., "all other things being equal"), meaning that two outcomes can be compared according to a variable only if these outcomes are equal over all other variables. Both dominance and undominated problems have been widely studied for this formalism. In general, dominance testing in CP-nets has been proved PSPACE-complete [3], whereas undominated outcomes are the solutions of a set of hard constraints, thus not harder than NP [4]. Dominance has been proved polynomial for CP-nets with tree or poly-tree structures, and for acyclic binary-valued CP-nets [5]. Undominated is also polynomial for acyclic CP-nets [5].

Several semantics other than ceteris paribus have been proposed: optimistic [6], pessimistic [7], strong (called strict in [8]). The conditional logic of preferences of [9], [10] provides a unified framework to deal with all these semantics. This framework has the advantage that it allows the user to choose a pessimistic or an optimistic semantics, and to decide for each preference whether it is strong or not. Unfortunately, the complexity of reasoning in this framework is unknown. The only algorithm provided for this framework requires exponential space.

In [11], Bienvenu et al. introduced another general framework for preference reasoning, called prototypical preference logic ( $P L$ for short). In $P L$, a logic formula involves preference statements. Each preference statement involves formulas $\alpha$ and $\beta$ and a formula $F$ under which $\alpha$ is preferred to $\beta$ (denoted by $\alpha \triangleright \beta \| F$ ). Several wellknown preference frameworks are fragments of $P L$. For instance, it has been shown in [11] that we can encode CPnets by putting in $F$ all the propositional symbols that do not appear in $\alpha$ and $\beta$. The expressive power of $P L$ has a price: dominance and consistency are shown to be PSPACEcomplete in the general case. In [11], Bienvenu et al. have also studied fragments with lower complexity. They isolated a part of $P L$ in which $F$ is empty and preferences only allow conjunctions. This fragment is called free preferences. With free preferences, consistency becomes co-NP-complete and dominance NP-complete. Free preferences are presented as "obviously related" to the strong semantics in [10].

In this paper, we study the optimistic/pessimistic preference logic defined in [9], [10]. We give a complete complexity map of the main problems in this logic: undominated, consistency and dominance. We show that their complexities depend on the semantics under which they are interpreted. All problems are NP-hard in general. When all preferences are strong, consistency and dominance are polynomial, and if in addition, the semantics is pessimistic, undominated is 
polynomial too. Then, we prove that the dominance problem, which has an online part in its definition, is compilable to polynomial time. After this compilation process, the dominance queries can be answered in linear time only, overcoming the practical difficulties imposed by the framework of [9], [10]. This result paves the way for using this framework in applications where we need to rank sets of outcomes on the fly.

\section{BACKGROUND}

We first define the vocabulary on which preferences will be defined. Intuitively, a vocabulary specifies the space $\Omega$ of all possible outcomes. A vocabulary $V$ is a pair $(X, D)$ such that $X$ is a set of $n$ variables $\left\{x_{1}, \ldots, x_{n}\right\}$, and $D$ is a set of finite domains $\left\{D\left(x_{1}\right), \ldots, D\left(x_{n}\right)\right\}$. An instantiation on a subset $X^{\prime} \subseteq X$ of variables is an assignment of a value of $D\left(x_{i}\right)$ to every variable $x_{i}$ in $X^{\prime}$. Instantiations on $X$ are called outcomes, that is, $\Omega=\Pi_{i \in 1 . . n} D\left(x_{i}\right)$. Given an outcome $I$ and an instantiation $u$ on $X^{\prime} \subseteq X$, we say that $I$ satisfies $u$ (denoted by $I \models u$ ) if the projection of $I$ on $X^{\prime}$ is equal to $u$. A preference network $N=(V, P)$ is defined by a set $P$ of $m$ preferences over the vocabulary $V=(X, D)$. $P$ is any preference formulation which allows to define an irreflexive and transitive binary relation $\succ$ over $\Omega$. For two outcomes $I$ and $I^{\prime}$ in $\Omega$, we say that $I$ is strictly preferred to $I^{\prime}$ when $I \succ I^{\prime}$.

\section{A. Main problems in preference reasoning}

The undominated, consistency, and dominance problems are the main problems defined in the literature for preference reasoning.

Definition 1 (Undominated): Given a preference network $N=(V, P)$, the undominated problem is to determine whether there exists an outcome $I \in \Omega$ for which there does not exist any outcome $I^{\prime} \in \Omega$ such that $I^{\prime} \succ I$.

Definition 2 (Consistency): Given a preference network $N=(V, P)$, the consistency problem is to determine whether there does not exist any pair of outcomes $I, I^{\prime} \in \Omega$ such that $I \succ I^{\prime}$ and $I^{\prime} \succ I$.

Definition 3 (Dominance): Given a preference network $N=(V, P)$ and two outcomes $I, I^{\prime} \in \Omega$, the dominance problem is to determine whether $I \succ I^{\prime}$ and $I^{\prime} \nsucc I$.

\section{B. Optimistic/pessimistic preference logic}

In this section, we present and formalize the conditional logic of preferences introduced in [9], [10]. This formalism can be used to build the relation $\succ$ over the outcomes in $\Omega$. The relation $\succ$ is induced by a set of preferences and semantics under which the preferences are interpreted. The user can define an optimistic (Opt) (resp. pessimistic $(P e s)$ ) semantics for the whole set of preferences, to rank the outcomes from best to worst (resp. from worst to best). After setting the semantics for the whole set of preferences, each preference can be defined either as strong (e.g., strong optimistic, strong pessimistic [8][12]), or non-strong (e.g., simply optimistic [13], [6] or pessimistic [7]). Illustrations of these semantics are given later. We give the definition of a preference in conditional logic.

Definition 4 (Preference): Given a vocabulary $V=$ $(X, D)$, a conditional logic preference $p$ is defined by a triplet $(h, b, w)$, written $p=(h: b>w)$, of instantiations in $V$. $h$ defines an hypothesis under which outcomes satisfying $b$ (better) are preferred to those satisfying $w$ (worse). If $h=\emptyset$ then $b$ is unconditionally preferred to $w$.

In the following we consider that in all preferences $p=$ $(h: b>w), h, b$, and $w$ are instantiations of bounded size. Despite this restriction, all our hardness results will hold.

Having defined what a preference in conditional logic is, we define the network of conditional logic preferences.

Definition 5 (CLP-net): A network of conditional logic preferences (CLP-net) is a pair $(V, P[S, S t r])$, where $V=$ $(X, D)$ is the vocabulary and $P$ is a set of conditional logic preferences defined on $V . S$ is a semantics for $P$ (i.e., $O p t$ or Pes), Str is the set of the preferences of $P$ that are strong.

Hereafter, when we refer to a preference, we always mean a conditional logic preference. We define two types of preference satisfaction, i.e. the satisfaction and the full satisfaction.

Definition 6 (Preference satisfaction): Given a preference $p=(h: b>w)$ on a vocabulary $V$, an outcome $I$ in $\Omega$ satisfies $p$ in $O p t$, written $I \models_{O p t} p$, if and only if $I \forall \neq h$ or $I \models b$ or $I \forall \neq w$. Respectively, $I$ satisfies $p$ in Pes, written $I \models_{P e s} p$, if and only if $I \not \models h$ or $I \not \models b$ or $I \models w$. If $I$ does not satisfy $p$ in $S$, we say that it violates $p$, written $I \not \not_{S} p$.

Given a CLP-net $(V, P[S, S t r])$ and an outcome $I$, $\operatorname{violated}(P, I)$ denotes the set of preferences from $P$ that are violated by $I$ in $S$. If $\operatorname{violated}(P, I)=\emptyset$, we write $I \models{ }_{S} P$.

An optimistic semantics $(S=O p t)$ can be viewed as a first best choice (namely the best outcome is given first), whereas a pessimistic semantics $(S=P e s)$ is a first worst choice (namely a choice by elimination).

We now give the definition for the preference full satisfaction, which requires to satisfy the hypothesis.

Definition 7 (Preference full satisfaction): Given a preference $p=(h: b>w)$ on a vocabulary $V$, an outcome $I$ in $\Omega$ fully satisfies $p$ in $O p t$, denoted by $\left.I\right|_{O p t} ^{f} p$, if and only if $I \models h, I \models b$, and $I \not \models w$. Respectively, I fully satisfies $p$ in Pes, denoted by $I \models{ }_{P e s}^{f} p$, if and only if $I \models h, I \models w$, and $I \not \forall b$.

We give an example to illustrate the preference (full) satisfaction.

Example 1 ((Full) satisfaction): When organizing a trip, there is an option between a day $(d)$ or a night $(\bar{d})$ flight 
and between a stop flight $(s)$ or a non-stop one $(\bar{s})$. The user says: "If the flight is non-stop then I prefer a night flight to a day one", written as: $p_{1}=(\bar{s}: \bar{d}>d)$. An outcome $I$ satisfies $p_{1}$ in $O p t$ if and only if $I$ violates $\bar{s}$ or satisfies $\bar{d}$. In other words, any stop flight or (non-stop) night flight satisfies user's preference in Opt. Similarly, any stop flight or (non-stop) day flight satisfies $p_{1}$ in Pes. An outcome $I$ fully satisfies $p_{1}$ in $O p t$ if and only if $I$ satisfies $\bar{s}$ and satisfies $\bar{d}$ (i.e., a non-stop night flight). A non-stop day flight fully satisfies $p_{1}$ in Pes.

Before defining the main notion that will allow us to compare outcomes, we need to define the notion of preference deactivation.

Definition 8 (Preference deactivation): Given a CLP-net $(V, P[S, S t r])$, we say that $P$ deactivates a preference $p$, denoted by $P \rightsquigarrow p$, if and only if

- $p \notin S t r$ and there exists an outcome $I \in \Omega$ such that $I$ fully satisfies $p$ in $S$ and $I$ satisfies $P$ in $S$, that is, $P \rightsquigarrow p \leftrightarrow \exists I \in \Omega|I|_{S}^{f} p$ and $I \models_{S} P$.

- $p \in S t r$ and for any outcome $I \in \Omega$, if $I$ fully satisfies $p$ in $S$ then $I$ satisfies $P$ in $S$, that is, $P \rightsquigarrow p \leftrightarrow \forall I \in$ $\Omega, I \models_{S}^{f} p \rightarrow I \models{ }_{S} P$.

Example 2 (Deactivation): In the problem of Example 1 where $p_{1}=(\bar{s}: \bar{d}>d)$, the user adds the following preference: "I prefer a non-stop flight to a stop one", written $p_{2}=(\emptyset: \bar{s}>s)$. Then, $P=\left\{p_{1}, p_{2}\right\}$. If the user sets $S$ to $O p t$ and $p_{1}, p_{2}$ are not strong, $P$ deactivates $p_{1}$ because the outcome $I=(\bar{s}, \bar{d})$ (i.e., "non-stop night flight") fully satisfies $p_{1}$ and satisfies $P$. $P$ also deactivates $p_{2}$.

Preference deactivation allows us to define layers, which is the central notion to compare outcomes in this conditional logic. A layer is a set of outcomes. Each layer includes the outcomes that do not belong to previous layers and that satisfy all preferences that have not been deactivated in previous layers. We give the inductive definition of layers.

Definition 9 (Layer): Given a CLP-net $(V, P[S, S t r])$, $P_{0}=P$, and $P_{i}$ is the set of the preferences in $P_{i-1}$ that are not deactivated by $P_{i-1}$, that is, $P_{i}=P_{i-1} \backslash\left\{p \mid P_{i-1} \rightsquigarrow p\right\}$. The layer $E_{i}$ is the set of outcomes that do not belong to previous layers and that satisfy all preferences in $P_{i}$, that is, $E_{i}=\left\{I \in \Omega \mid I \models_{S} P_{i}\right\} \backslash \bigcup_{j \in 0 . . i-1} E_{j}$. The index last is the smallest index such that all outcomes satisfying $P_{\text {last }}$ belong to previous layers $E_{0}, \ldots, E_{\text {last-1 }}$. We define $E_{\text {last }}$ to be the set of remaining outcomes $\Omega \backslash \bigcup_{j \in 0 . . \text { last }-1} E_{j}$.

Given an outcome $I \in \Omega$, layer $(I)$ is the index of the layer to which $I$ belongs, that is, $I \in E_{\text {layer }(I)}$.

It is important to observe that $E_{\text {last }}$ can be empty or not. If $E_{\text {last }}$ is non-empty, none of the outcomes in $E_{\text {last }}$ can satisfy $P_{\text {last }}$ and the construction of layers cannot proceed.

We define now the preference relation $\succ$ according to the semantics used in CLP-nets.
Table I

THE RANKINGS OF EXAMPLE 3

\begin{tabular}{c|c|c||c|c|} 
& \multicolumn{2}{|c||}{$p_{1}, p_{2} \notin S t r$} & \multicolumn{2}{c|}{$p_{1} \notin S t r, p_{2} \in S t r$} \\
\hline$i$ & $P_{i}$ & $E_{i}$ & $P_{i}$ & $E_{i}$ \\
\hline 0 & $p_{1}, p_{2}$ & $(\bar{s}, \bar{d})$ & $p_{1}, p_{2}$ & $(\bar{s}, \bar{d})$ \\
\hline 1 & $\emptyset$ & $(s, \bar{d}),(s, d),(\bar{s}, d)$ & $p_{2}$ & $(\bar{s}, d)$ \\
\hline 2 & $\emptyset$ & $\emptyset$ & $\emptyset$ & $(s, d),(s, \bar{d})$ \\
\hline 3 & - & - & $\emptyset$ & $\emptyset$ \\
\hline
\end{tabular}

Definition 10 (Layer based order): Given two outcomes $I$ and $I^{\prime}$ in $\Omega$, we have,

- $I$ is strictly preferred to $I^{\prime}$ in $O p t$, denoted by $I \succ_{O p t}$ $I^{\prime}$, if and only if layer $\left(I^{\prime}\right)=$ last or layer $(I)<$ layer $\left(I^{\prime}\right)$.

- $I$ is strictly preferred to $I^{\prime}$ in Pes, denoted by $I \succ_{P e s}$ $I^{\prime}$, if and only if layer $(I)=$ last or layer $(I)>$ layer $\left(I^{\prime}\right)$.

When $S=O p t$ (resp. $S=P e s$ ), the best (resp. worst) outcome is ranked first. Namely, the best outcome appears in $E_{0}$ for $O p t$ (resp. in $E_{\text {last-1 }}$ for Pes). If an outcome $I$ belongs to a layer which precedes (resp. follows) the layer where an outcome $I^{\prime}$ belongs to, $I$ is strictly preferred to $I^{\prime}$.

Example 3 (Layers): Recall the preferences of the previous examples: $p_{1}=(\bar{s}: \bar{d}>d)$ and $p_{2}=(\emptyset: \bar{s}>s)$. Table I illustrates the different rankings of the outcomes depending on $p_{2}$ being strong or not. Suppose that $p_{1} \notin S t r$ and $p_{2} \notin S t r$. According to Definition 6, the best outcome for $S=O p t$ is the same regardless of the set $S t r$, i.e. a non-stop night flight $(\bar{s}, \bar{d})$. Hence, $E_{0}=\{(\bar{s}, \bar{d})\}$. From Definitions 7, 8 and 9 , if $p_{1} \notin S t r$ and $p_{2} \notin S t r$ then $P_{0}=P=\left\{p_{1}, p_{2}\right\}$ deactivates both $p_{1}$ and $p_{2}$. Indeed, $(\bar{s}, \bar{d})$ satisfies $P_{0}$ and fully satisfies $p_{1}$ and $p_{2}$. Therefore, the set of outcomes in $E_{1}$ which satisfy $P_{1}=P_{0} \backslash\left\{p_{1}, p_{2}\right\}=\emptyset$ is equal to all remaining outcomes, namely $E_{1}=\{(s, \bar{d}),(s, d),(\bar{s}, d)\}$. $E_{2}=\emptyset$ and last $=2$.

Suppose now that $p_{1} \notin S t r$ and $p_{2} \in S t r$. $P_{0}$ deactivates $p_{1}$ due to $(\bar{s}, \bar{d})$, but does not deactivate $p_{2}$ because $p_{2} \in S t r$ and there exists $I^{\prime}=(\bar{s}, d)$ such that $I^{\prime}$ fully satisfies $p_{2}$ but violates $p_{1}$. Thus, $E_{0}=\{(\bar{s}, \bar{d})\}$ and $P_{1}=P_{0} \backslash\left\{p_{1}\right\}=\left\{p_{2}\right\} .(\bar{s}, \bar{d})$ and $(\bar{s}, d)$ satisfy $p_{2}$, resulting in $E_{1}=\{(\bar{s}, \bar{d}),(\bar{s}, d)\} \backslash E_{0}=\{(\bar{s}, d)\}$. The outcomes that fully satisfy $p_{2}$ (i.e., $(\bar{s}, \bar{d})$ and $(\bar{s}, d)$ ) also satisfy $P_{1}$. Thus, $P_{1}$ deactivates $p_{2} . P_{2}=\emptyset, E_{2}=\{(s, d),(s, \bar{d})\}$, and last $=3$.

We have shown how a preference is interpreted under its semantics. In the following section, we show the complexity of the three problems undominated/consistency/dominance depending on the semantics.

\section{COMPLEXITY MAP}

In this section we draw the complexity map for the three problems undominated/consistency/dominance. We first fo- 
cus on the optimistic semantics and then adapt the results to the pessimistic case.

\section{A. Optimistic semantics}

Theorem 1: The undominated problem is NP-complete on CLP-nets with $S=O p t$, even if $S t r=P$.

Proof: Membership. Given an outcome $I \in \Omega$, checking whether $I$ is an undominated outcome in $O p t$ is equivalent to checking if $I$ belongs to $E_{0}$ and last $\neq 0$. (Outcomes in $E_{\text {last }}$ are all preferred one to each other (Definition 10). Thus, no outcome in $E_{\text {last }}$ can be an undominated outcome.) Following Definition $9, I$ belongs to $E_{0}$ and last $\neq 0$ if and only if $I$ satisfies all $p \in P$ in Opt. $I$ satisfies a preference $p=(h: b>w)$ in Opt if and only if $I$ violates the hypothesis $h$ or $w$ or satisfies $b$. Checking if $I$ violates $h$ or $w$ or if $I$ satisfies $b$ is polynomial and this verification is performed exactly $|P|$ times.

Completeness. We reduce an instance $\phi=\left(c l_{1}, \ldots c l_{m}\right)$ of 3-SAT to the undominated problem: the set of variables $X$ is defined by variables $(\phi)$ and $D$ is $\{0,1\}^{X} . P$ is built as follows. For each clause $c l_{i}=\left(l_{i, 1}, l_{i, 2}, l_{i, 3}\right)$ of $\phi$, we introduce a preference $p_{i}=\left(h_{i}: b_{i}>w_{i}\right)$ with $h_{i}=\neg l_{i, 1}$, $b_{i}=l_{i, 2}$ and $w_{i}=\neg l_{i, 3}$. An outcome $I$ satisfies a preference $p_{i}$ if and only if $I$ violates $h_{i}$ or $w_{i}$ or satisfies $b_{i}$. Hence, $I$ satisfies $p_{i}$ if and only if it satisfies $l_{i, 1}$ or $l_{i, 2}$ or $l_{i, 3}$, that is, it satisfies the clause $c l_{i}$. Now, there exists an undominated outcome if and only if there exists an outcome $I$ that belongs to $E_{0}$, last $\neq 0$. I belongs to $E_{0}$ with last $\neq 0$ if and only if $I$ satisfies $P$, and $I$ satisfies $P$ if and only if it satisfies all clauses in $\phi$.

The proof still holds if $S t r=P$ because the satisfaction of a preference $p$ is the same whatever $p$ is in $S t r$ or not.

To prove the complexity of the consistency and dominance problems we will use the following lemmas.

Lemma 1: Given a CLP-net $N$ with $S=O p t$, these three propositions are equivalent:

(1) $N$ is consistent,

(2) $E_{\text {last }}$ is empty,

(3) $P_{\text {last }}$ is empty.

Proof: $((1) \Rightarrow(3))$ We show that if $P_{\text {last }}$ is not empty, then $N$ is inconsistent. Let $p$ be a preference in $P_{\text {last }}$. There necessarily exists an outcome $I$ in $\Omega$ violating $p$. $I$ cannot be in $\bigcup_{i \in 0 \text {..last-1 }} E_{i}$ as $p$ is still active in $P_{\text {last }}$. So, $I \in E_{\text {last }}$. There also necessarily exists an outcome $I^{\prime}$ in $\Omega$ that fully satisfies $p$. Again, $I^{\prime}$ cannot be in $\bigcup_{i \in 0 . \text { last }-1} E_{i}$, otherwise $p$ would have been deactivated and would not be in $P_{l a s t}$. Thus, both $I$ and $I^{\prime}$ belong to $E_{\text {last }} . I^{\prime}$ cannot be equal to $I$ because $I^{\prime}$ satisfies $p$ whereas $I$ does not, and by Definition 10, we know that for any $I, I^{\prime}$ in $E_{\text {last }}, I \succ I^{\prime}$ and $I^{\prime} \succ I$. Therefore, $N$ is inconsistent.

$((3) \Rightarrow(2))$ Assume $E_{\text {last }}$ is not empty. By Definition 9, $E_{\text {last }}$ contains those outcomes that do not satisfy $P_{\text {last }}$.
Therefore, $P_{\text {last }}$ is not empty because any outcome satisfies an empty set of preferences.

$((2) \Rightarrow(1))$ Assume $E_{\text {last }}$ is empty. From Definition 10, there exists a pair of outcomes $I, I^{\prime}$ such that $I \succ I^{\prime}$ and $I^{\prime} \succ I$ if and only if both $I$ and $I^{\prime}$ belong to $E_{\text {last }}$. Since $E_{\text {last }}$ is empty, there does not exist such a pair of outcomes and then $N$ is consistent.

Lemma 2: Given a CLP-net $N=(V, P[O p t, S t r])$, deciding whether a preference $p$ is deactivated by a set $P_{i} \subseteq P$ is polynomial if $p \in S t r$.

Proof: By definition of deactivation (Definition 8), $P_{i}$ deactivates a strong preference $p=(h: b>w)$ if and only if there does not exist any outcome $I$ that fully satisfies $p$ and violates a preference $p^{\prime}=\left(h^{\prime}: b^{\prime}>w^{\prime}\right)$ in $P_{i}$. Testing whether there exists $I$ such that $I$ fully satisfies $p$ and violates $p^{\prime}$ is equivalent to testing whether there exists $I$ satisfying $h \wedge b \wedge \neg w \wedge h^{\prime} \wedge \neg b^{\prime} \wedge w^{\prime}$. Such a test is linear in the number of variables $n$ as all terms of the conjunction are instantiations. We do this process for each $p^{\prime}$ in $P_{i}, p^{\prime} \neq p$. $P_{i}$ deactivates $p$ if and only if all the $\left|P_{i}\right|-1$ tests return false. Therefore, deciding deactivation of a strong preference is polynomial in Opt.

Theorem 2: The consistency problem is NP-complete on CLP-nets with $S=O p t$.

Proof: By Lemma 1, a CLP-net with $S=O p t$ is consistent if and only if $P_{l a s t}$ is empty.

Membership. Let us first observe that if a set $Q$ of preferences deactivates a preference $p$, then any subset of $Q$ also deactivates $p$. A sequence of preferences $\left\langle p_{i_{1}}, \ldots, p_{i_{k}}\right\rangle$ such that $\forall j \in 1 . . k, P \backslash\left\{p_{i_{l}} \mid l<j\right\}$ deactivates $p_{i_{j}}$ is called sequence of deactivations. Given a sequence of deactivations $\left\langle p_{i_{1}}, \ldots, p_{i_{j}}, \ldots, p_{i_{k}}\right\rangle$, if $P \backslash\left\{p_{i_{1}}, \ldots, p_{i_{j}}\right\}$ deactivates $p_{i_{k}}$, then $\left\langle p_{i_{1}}, \ldots, p_{i_{j}}, p_{i_{k}}, \ldots, p_{i_{k-1}}\right\rangle$ is also a sequence of deactivations because of our first observation. Hence, a polynomial certificate for $P_{\text {last }}=\emptyset$ is any sequence of deactivations that wipes out $P$. Let $S e q$ be the sequence $\left\langle\left\langle p_{i_{1}}, I_{i_{1}}\right\rangle, \ldots,\left\langle p_{i_{m}}, I_{i_{m}}\right\rangle\right\rangle$ such that $P=\left\{p_{i_{1}}, \ldots, p_{i_{m}}\right\}$, $p_{i_{k}}$ is deactivated by $P \backslash\left\{p_{i_{j}} \mid j<k\right\}$ for all $k \in 1 . . m$, and for each $p_{i_{k}} \notin S t r, I_{i_{k}}$ is an outcome that fully satisfies $p_{i_{k}}$ and that satisfies $P \backslash\left\{p_{i_{j}} \mid j<k\right\}$. If $p_{i_{k}} \in S t r$, checking if $p_{i_{k}}$ is deactivated by $P \backslash\left\{p_{i_{j}} \mid j<k\right\}$ is polynomial by Lemma 2. If $p_{i_{k}} \notin S t r$, checking if $p_{i_{k}}$ is deactivated by $P \backslash\left\{p_{i_{j}} \mid j<k\right\}$ is equivalent to checking if $I_{i_{k}}$ satisfies $P \backslash\left\{p_{i_{j}} \mid j<k\right\}$ and fully satisfies $p_{i_{k}}$, which is also polynomial. Thus, $S e q$ is a polynomial certificate for the consistency problem.

Completeness. We reduce an instance $\phi=\left(c l_{1}, \ldots c l_{m-1}\right)$ of 3-SAT to the problem $E_{\text {last }}=\emptyset$ in the CLP-net $(V, P[O p t, S t r])$. The set of variables $X$ is defined by variables $(\phi) \cup\left\{l_{b}\right\}$ and $D=\{0,1\}^{X}$. For each clause $c l_{i}=\left(l_{i, 1}, l_{i, 2}, l_{i, 3}\right)$ of $\phi$, we add to $P$ a preference $p_{i}=\left(\emptyset: l_{b}>\neg l_{i, 1} \wedge \neg l_{i, 2} \wedge \neg l_{i, 3}\right)$, and $p_{i} \notin S t r$. We add to $P$ an extra preference $p_{\text {extra }}=\left(\emptyset: \neg l_{b}>l_{b}\right)$ with 
$p_{\text {extra }} \notin S t r$. We prove that $\phi$ is satisfiable if and only if $E_{\text {last }}=\emptyset$.

$(\Rightarrow)$ Let $I_{\phi}$ be a model of $\phi$. By construction, $I_{\phi} \cup\left\{l_{b}=0\right\}$ satisfies all preferences in $P$ and fully satisfies $p_{\text {extra }}$. No outcome can satisfy $p_{\text {extra }}$ and fully satisfy another preference in $P$. Hence, $P_{1}=P \backslash\left\{p_{\text {extra }}\right\}$. By construction again, $I_{\phi} \cup\left\{l_{b}=1\right\}$ fully satisfies all preferences in $P_{1}$, which leads to $P_{2}=\emptyset$ and $E_{3}=E_{\text {last }}=\emptyset$.

$(\Leftarrow)$ Assume $E_{\text {last }}$ is empty. Then, last cannot be equal to 0 because by definition, $E_{0}$ cannot be empty. Thus, $E_{0}$ contains at least an outcome $I$, and by construction $I$ satisfies $P$, as last $\neq 0$. To satisfy $p_{\text {extra }} I$ must violate $l_{b}$. Thus, $I$ satisfies $P$ if and only if for all $p_{i}$ in $P, I$ violates $\neg l_{i, 1} \wedge \neg l_{i, 2} \wedge \neg l_{i, 3}$, and thus satisfies $c l_{i}$. Therefore, $I$ satisfies $\phi$.

Theorem 3: The dominance problem is DP-complete on CLP-nets with $S=O p t$.

Proof: Membership. Let $N=(V, P[O p t, S t r])$ be a CLP-net and $I, I^{\prime}$ two outcomes. A certificate for the dominance problem is a certificate for the problem of the existence of an integer $j$ such that $I \in$ $\bigcup_{i \in 0 . . j} E_{i}$ and $I^{\prime} \notin \bigcup_{i \in 0 . . j} E_{i}$. Let $S_{j}=\left\langle\left\langle p_{1}^{1}, I_{1}^{1}\right\rangle, \ldots\right.$, $\left.\left\langle p_{i_{1}}^{1}, I_{i_{1}}^{1}\right\rangle, \ldots,\left\langle p_{1}^{j}, I_{1}^{j}\right\rangle, \ldots,\left\langle p_{i_{j}}^{j}, I_{i_{j}}^{j}\right\rangle\right\rangle$ be a sequence of deactivations such that each $\left\langle p_{y}^{x}, I_{y}^{x}\right\rangle \in S_{j}$ has the following properties: (1) $P \backslash\left\{p_{b}^{a} \mid\left\langle p_{b}^{a}, I_{b}^{a}\right\rangle \in S_{j} \wedge a<x\right\} \rightsquigarrow p_{y}^{x}$, (2) if $p_{y}^{x} \notin S t r, I_{y}^{x}$ is a witness of the deactivation in (1), and (3) $\operatorname{violated}(P, I) \subseteq\left\{p_{y}^{x} \mid\left\langle p_{y}^{x}, I_{y}^{x}\right\rangle \in S_{j}\right\}$. Let $S_{j}^{\prime}=\left\langle\left\langle p_{1}^{\prime 1}, I_{1}^{\prime 1}\right\rangle\right.$, $\left.\ldots,\left\langle p_{i_{1}^{\prime}}^{\prime 1}, I_{i_{1}^{\prime}}^{\prime 1}\right\rangle, \ldots,\left\langle p_{1}^{\prime j}, I_{1}^{\prime j}\right\rangle, \ldots,\left\langle p_{i_{j}^{\prime}}^{\prime j}, I_{i_{j}^{\prime}}^{\prime j}\right\rangle\right\rangle$ be a sequence of deactivations built in a way similar to $S_{j}$, that is, for each $\left\langle p_{y}^{\prime x}, I_{y}^{\prime x}\right\rangle \in S_{j}^{\prime}$, (1) $P \backslash\left\{p_{b}^{\prime a} \mid\left\langle p_{b}^{\prime a}, I_{b}^{\prime a}\right\rangle \in S_{j}^{\prime} \wedge a<x\right\}$ $\rightsquigarrow p_{y}^{\prime x}$, (2) if $p_{y}^{\prime x} \notin S t r, I_{y}^{\prime x}$ is a witness of the deactivation of $p_{y}^{\prime x}$, and (3) violated $\left(P, I^{\prime}\right) \subseteq\left\{p_{y}^{\prime x} \mid\left\langle p_{y}^{\prime x}, I_{y}^{\prime x}\right\rangle \in S_{j}^{\prime}\right\}$.

$S_{j}$ is a certificate of $I \in \bigcup_{i \in 0 . . j} E_{i}$ and $S_{j}^{\prime}$ is a certificate of $I^{\prime} \in \bigcup_{i \in 0 . . j} E_{i}$. They are both polynomial to check: for each pair $\langle p, I\rangle$ in $S_{j}$ and $S_{j}^{\prime}$, if $p \in S t r$ we know by Lemma 2 that deactivation is polynomial, and if $p \notin S t r$, we check whether $I$ fully satisfies $p$ and satisfies the set of preferences specified in (1). Finally, we check that all preferences in violated $(P, I)$ (resp. violated $\left(P, I^{\prime}\right)$ ) are covered. As dominance says 'yes' if and only if there exists such an $S_{j}$ and there does not exist such an $S_{j}^{\prime}$, we conclude that dominance is in $D P$.

Completeness. We reduce an instance of SAT-UNSAT to the dominance problem. Let $F=\left(c l_{1}, \ldots, c l_{|F|}\right)$ and $F^{\prime}=\left(c l_{1}^{\prime}, \ldots, c l_{\left|F^{\prime}\right|}^{\prime}\right)$ be the two 3-CNF formulas of the SAT-UNSAT instance. We build the CLP-net $((X, D), P[O p t, S t r])$, and two outcomes $I$ and $I^{\prime}$, as an instance of the dominance problem. $X$ is the union of variables $(F)$ and variables $\left(F^{\prime}\right)$ plus four additional variables $\left\{l_{h}, l_{h^{\prime}}, l_{w}, l_{w^{\prime}}\right\}$, and $D=\{0,1\}^{X}$. For each clause $c l_{i}=\left(l_{i, 1}, l_{i, 2}, l_{i, 3}\right)$ of $F$, we define a preference $p_{i}=\left(l_{h}: l_{i, 1}>\neg l_{i, 2} \wedge \neg l_{i, 3}\right)$. For each clause $c l_{i}^{\prime}=$ $\left(l_{i, 1}^{\prime}, l_{i, 2}^{\prime}, l_{i, 3}^{\prime}\right)$ of $F^{\prime}$, we define a preference $p_{i}^{\prime}=\left(l_{h^{\prime}}\right.$ : $\left.l_{i, 1}^{\prime}>\neg l_{i, 2}^{\prime} \wedge \neg l_{i, 3}^{\prime}\right)$. We introduce two additional preferences $p=\left(\emptyset: l_{h}>l_{w}\right)$ and $p^{\prime}=\left(\emptyset: l_{h^{\prime}}>l_{w^{\prime}}\right)$. We then have, $P=\left\{p_{1}, \ldots, p_{|F|}, p_{1}^{\prime}, \ldots, p_{\left|F^{\prime}\right|}^{\prime}, p, p^{\prime}\right\}$, and we set $S t r=\emptyset$. We define $I$ as an arbitrary outcome over $X$ satisfying $\neg l_{h} \wedge l_{w} \wedge \neg l_{h^{\prime}} \wedge \neg l_{w^{\prime}}$. Similarly, we define $I^{\prime}$ as an arbitrary outcome over $X$ satisfying $\neg l_{h} \wedge \neg l_{w} \wedge \neg l_{h^{\prime}} \wedge l_{w^{\prime}}$.

We now prove that $I$ dominates $I^{\prime}$ (i.e., $I \succ I^{\prime}$ and $I^{\prime} \nsucc I$ ) if and only if $F$ is SAT and $F^{\prime}$ is UNSAT. We first prove that $F$ is SAT if and only if $I$ belongs to $E_{1} . I$ violates $p$ because $I \models \neg l_{h} \wedge l_{w}$. Hence, $I$ cannot belong to $E_{0}$. By construction, $I$ satisfies all other preferences in $P$. Thus, $I$ belongs to $E_{1}$ if and only if $P$ deactivates $p$, which means that there exists an outcome $I^{*}$ such that $I^{*}$ satisfies $P$ and fully satisfies $p$. If we set $l_{w^{\prime}}$ and $l_{h^{\prime}}$ to false in $I^{*}, I^{*}$ satisfies $p^{\prime}$ and all $p_{i}^{\prime}$. To fully satisfy $p, I^{*}$ needs to satisfy $l_{h} \wedge \neg l_{w}$. As $I^{*} \models l_{h}$, to satisfy any $p_{i}$ (obtained from a $c l_{i}$ of $\left.F\right), I^{*}$ needs to satisfy $l_{i, 1} \vee \neg\left(\neg l_{i, 2} \wedge \neg l_{i, 3}\right)$, namely $\left(l_{i, 1} \vee l_{i, 2} \vee l_{i, 3}\right)$. Since $I^{*}$ satisfies all these $p_{i}, I^{*}$ is a model of $F$. Then $I$ belongs to $E_{1}$ if and only if $F$ is SAT.

Second, we prove that $I^{\prime}$ does not belong to $E_{1}$ if and only if $F^{\prime}$ is UNSAT. $I^{\prime}$ violates $p^{\prime}$ because $I^{\prime} \models \neg l_{h^{\prime}} \wedge l_{w^{\prime}}$. Hence, $I^{\prime}$ cannot belong to $E_{0} . I^{\prime}$ does not belong to $E_{1}$ if and only if $P$ does not deactivate $p^{\prime}$, which means that there does not exist an outcome $I^{\prime *}$ such that $I^{\prime *}$ satisfies $P$ and fully satisfies $p^{\prime}$. By using the same reasoning as previously, there does not exist such an $I^{\prime *}$ if and only if $F^{\prime}$ is UNSAT. Thus $I^{\prime}$ does not belong to $E_{1}$ if and only if $F^{\prime}$ is UNSAT.

Finally, we observe that if $F$ and $F^{\prime}$ are both UNSAT, neither $p$ nor $p^{\prime}$ can be deactivated, so $I$ and $I^{\prime}$ both belong to $E_{2}$, which is equal to $E_{\text {last }}$, and $I$ does not dominate $I^{\prime}$.

Theorem 1 tells us that the undominated problem is NPcomplete for $S=O p t$ even if $S t r=P$. Interestingly, this is not the case for the consistency and dominance problems, which become polynomial when $S t r=P$.

Theorem 4: The consistency problem is polynomial on CLP-nets with $S=O p t$ and $S t r=P$.

Proof: A CLP-net $(V, P[O p t, S t r])$ is consistent if and only if $P_{\text {last }}$ is empty (Lemma 1). This means that there exists a sequence of deactivations that wipes out $P$, that is, a sequence of preferences $\left\langle p_{i_{1}}, \ldots, p_{i_{m}}\right\rangle$ such that $P=\left\{p_{i_{j}} \mid\right.$ $j \in 1 . . m\}$ and for all $k \in 1 . . m, P \backslash\left\{p_{i_{j}} \mid j<k\right\} \rightsquigarrow p_{i_{k}}$. By Lemma 2 , deciding the deactivation of a strong preference by a set of preferences is polynomial. Thus, given a set $Q$ of strong preferences, finding whether there exists a preference in $Q$ deactivated by $Q$ is polynomial too. It is sufficient to iteratively check every preference in $Q$. Next, as in the proof of Theorem 2, we observe that if $Q$ deactivates a preference $p$, then any subset of $Q$ also deactivates $p$. Thus, there exits a sequence of deactivations that wipes out $P$ if and only if a greedy algorithm iteratively finding deactivated preferences and removing them from $P$ terminates by wiping out $P$. As a result, if $P$ can be wiped out by this process, the CLP-net 
is consistent, otherwise it is inconsistent. In the worst case, finding a preference that is deactivated by $P$ or by a subset of $P$ requires $|P|$ calls to the deactivation problem. This search for deactivated preference is performed $|P|$ times. Therefore, the consistency problem is polynomial.

Theorem 5: The dominance problem is polynomial on CLP-nets with $S=O p t$ and $S t r=P$.

Proof: (Sketch.) Given a CLP-net $(V, P[O p t, S t r])$ and two outcomes $I$ and $I^{\prime}$, dominance says 'yes' if and only if there exists an integer $j$ such that $I \in \bigcup_{i \in 0 . . j} E_{i}$ and $I^{\prime} \notin \bigcup_{i \in 0 . . j} E_{i}$. We can design an algorithm that iteratively builds the sets of preferences $P_{1}, \ldots, P_{j}$, where $P_{i}$ is the set of preferences not deactivated by $P_{i-1}$ and $j$ is the smallest integer such that $I \models{ }_{O p t} P_{j} . P_{j}$ is such that $I \succ I^{\prime}$ if and only if $I^{\prime} \not{ }_{O p t} P_{j}$.

Building $P_{1}, \ldots, P_{j}$ is polynomial because deciding the deactivation of a strong preference by a set of preferences is polynomial (Lemma 2) and the number of deactivation tests is bounded above by $|P|^{2}$. Finally, checking whether $I^{\prime}$ satisfies $P_{j}$ is again polynomial to check.

\section{B. Pessimistic semantics}

As seen in Definition 6, the pessimistic semantics ranks first layers of the worst outcomes whereas optimistic semantics ranks first layers of the best outcomes. As a result, most of the proofs given in this section are slight adaptations of previous ones.

Theorem 6: The consistency problem is NP-complete on CLP-nets with $S=P e s$.

Proof: (Sketch.) Membership is essentially the same as in the proof of Theorem 2. Completeness is obtained by changing the way we encode clauses in the proof of Theorem 2: For each clause $c l_{i}=\left(l_{i, 1}, l_{i, 2}, l_{i, 3}\right)$ of $\phi$, we introduce a preference $p_{i}=\left(\emptyset: \neg l_{i, 1} \wedge \neg l_{i, 2} \wedge \neg l_{i, 3}>l_{b}\right)$ instead of $p_{i}=\left(\emptyset: l_{b}>\neg l_{i, 1} \wedge \neg l_{i, 2} \wedge \neg l_{i, 3}\right)$. The extra preference becomes $p_{\text {extra }}=\left(\emptyset: l_{b}>\neg l_{b}\right)$.

Theorem 7: The dominance problem is $D P$-complete on CLP-nets with $S=P e s$.

Proof: Direct adaptation of the proof of Theorem 3.

The undominated problem in pessimistic semantics is substantially different from the optimistic case as we have to build the whole sequence of layers from worst to best before proving the existence of an undominated outcome. It follows a strong connection between the consistency and undominated problems.

Lemma 3: A CLP-net with $S=P e s$ is consistent if and only if it has an undominated outcome.

Proof: $(\Rightarrow)$ Let $N$ be a consistent CLP-net with $S=$ Pes. From a slightly adapted Lemma $1, N$ is consistent if and only if $E_{\text {last }}$ is empty. As $E_{\text {last }}$ is empty, there exists an outcome $I$ belonging to $E_{\text {last-1 }}$. By Definition 10, there does not exist any outcome $I^{\prime}$ such that $I^{\prime} \succ I$. Thus, $I$ is undominated.

$(\Leftarrow)$ Assume $N$ is inconsistent. Then, there exist two outcomes $I$ and $I^{\prime}$ such that $I \succ I^{\prime}$ and $I^{\prime} \succ I$. According to Definition 10, it can only happen if $I$ and $I^{\prime}$ both belong to $E_{\text {last }}$. Suppose there exists an undominated outcome $I^{*} . I^{*}$ necessarily belongs to $E_{\text {last }}$, but it is impossible as $I$ would dominate it.

Corollary 1: The undominated problem is NP-complete on CLP-nets with $S=P e s$.

\section{Proof: From Lemma 3 and Theorem 6.}

Theorem 8: The undominated/consistency/dominance problems are polynomial on CLP-nets with $S=P e s$ and Str $=P$.

Proof: (Sketch.) Consistency and dominance are similar to the optimistic case (Theorems 4 and 5). Undominated comes from Lemma 3.

Table II summarizes the complexity results of this section.

Table II

SUMMARY OF OUR COMPLEXITY RESULTS

\begin{tabular}{|l|c|c|c|}
\hline Problem & Str & $S=O p t$ & $S=$ Pes \\
\hline \multirow{2}{*}{ Undominated } & $\mp P$ & NP-complete (Th. 1) & NP-complete (Co. 1) \\
\cline { 2 - 4 } & $=P$ & NP-complete (Th. 1) & polynomial (Th. 8) \\
\hline \multirow{2}{*}{ Consistency } & $\varsubsetneqq P$ & NP-complete (Th. 2) & NP-complete (Th. 6) \\
\cline { 2 - 4 } & $=P$ & polynomial (Th. 4) & polynomial (Th. 8) \\
\hline \multirow{2}{*}{ Dominance } & $\mp P$ & $D P$-complete (Th. 3) & DP-complete (Th. 7) \\
\cline { 2 - 4 } & $=P$ & polynomial (Th. 5) & polynomial (Th. 8) \\
\hline
\end{tabular}

\section{SOlving AND COMPILING CLP-NETS}

The three problems undominated/consistency/dominance introduced in Section II-A are NP-hard to solve in general. The undominated and consistency problems are static problems belonging to NP. They can be solved by a simple call to a SAT or CSP solver. The dominance problem, on the contrary, is more difficult for two reasons. First, it is beyond NP. But more importantly, dominance queries can be repeatedly asked on the same CLP-net for comparing different pairs of outcomes. Such queries arise for instance in recommendation applications, where the system/provider wants to propose to the customer the most appropriate outcome among two (or more) outcomes, according to her preferences. As these queries can be involved in an interactive process, it is crucial to have fast responses.

Compilation theory aims at improving the efficiency of on-line computation of difficult problems through preprocessing [14], [15]. The intuition is to remove sources of complexity to obtain cheaper queries (belonging to a lower complexity class) using a compiled form of the initial data $\Sigma$. The pre-processing, or off-line compilation phase, consists in transforming parts of this fixed part $\Sigma$ into a compiled form of size polynomial in $|\Sigma|$. This transformation can take exponential time. The varying part of the problem appears at execution time, called on-line phase. If the output can be 
produced in polynomial time, then the problem is said to be compilable to polynomial time. We show that dominance queries become polynomial after a compilation phase that produces an array index $P[\cdot]$ of indices, where $i n d e x P[j]$ is the index of the first layer where $p_{j}$ is not active, that is, index $P[j]=i$ if and only if $p_{j} \in P_{i-1} \backslash P_{i}$.

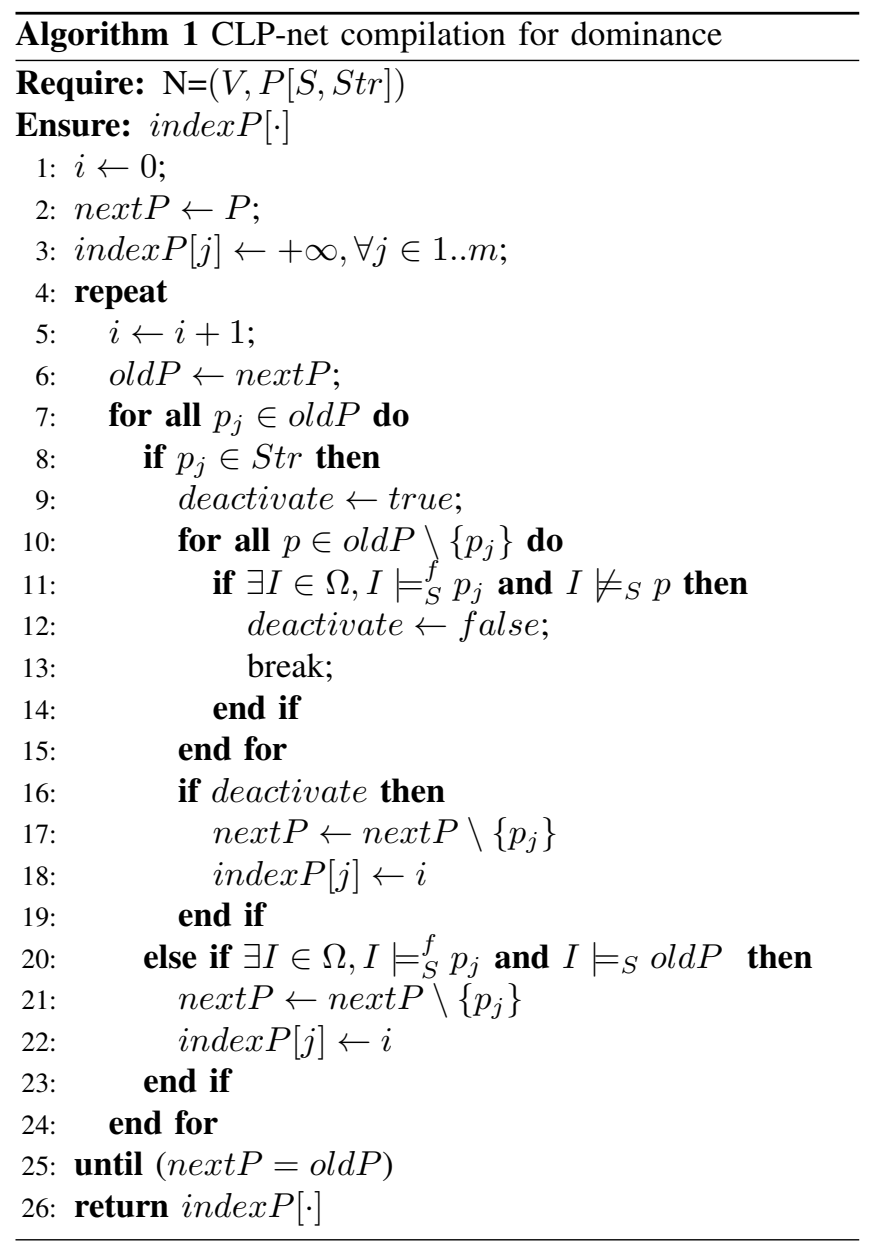

Algorithm 1 takes as input a CLP-net $(V, P[S, S t r])$ and returns the array index $P[\cdot]$. In line 3 index $P[\cdot]$ is initialized to a value greater than any possible layer. Then, the loop of line 4 iteratively builds index $P[\cdot]$. The $i$ th execution of the loop sets oldP to the set of preferences still active at the previous layer $i-1$ (line 6) and will compute the set next $P$ of preferences still active at layer $i$. The loop of line 7 iterates over all preferences $p_{j}$ that belong to oldP to check if $p_{j}$ can be deactivated. The deactivation depends on whether the semantics of $p_{j}$ is strong or not. If $p_{j} \in S t r$ (lines 8-19), we need to iterate over all other preferences $p$ in oldP. If there exists an $I$ fully satisfying $p_{j}$ and violating $p$ (line 11), flag deactivate becomes false (line 12) and we proceed to the next preference in oldP. If such an $I$ is not found, then $p_{j}$ is deactivated (that is, removed from next $P$ ), and $i n \operatorname{dex} P[j]$ is set to $i$ (lines 16-19). When $p_{j} \notin S t r$ (lines 20-22), we need to check if there exists an outcome $I$ that fully satisfies $p_{j}$ and satisfies all preferences in oldP. This line is NP-complete and can be solved by a call to a SAT/CSP solver. If such an outcome is found, again $p_{j}$ is deactivated (removed from next $P$ ) and $i n d e x P[j]$ is set to $i$ (lines 21-22). Finally the test of the main loop (in line 4) detects whether at least one preference has been deactivated from one layer to another. If not, we exit the loop and return index $P[\cdot]$.

Theorem 9: The Dominance problem in CLP-nets is compilable to linear time.

Proof: A problem is compilable to a complexity class $C$ if it is in $C$ once the fixed part $\Sigma$ of any instance has been pre-processed, i.e. turned off-line into a data structure of size polynomial in $|\Sigma|$. Given $(V, P[S, S t r])$, Algorithm 1 computes off-line the array index $P[\cdot]$, where $i n d \operatorname{ex} P[j]=i$ if and only if $p_{j} \in P_{i-1} \backslash P_{i}$. This structure $i n \operatorname{dex} P[\cdot]$ is linear in $|P|$, the initial data.

Correctness. We iterate over violated $(P, I)$ to compute $i=\max _{p_{j} \in \operatorname{violated}(P, I)}$ index $P[j]$ and over violated $\left(P, I^{\prime}\right)$ to compute $i^{\prime}=\max _{p_{j} \in \operatorname{violated}\left(P, I^{\prime}\right)}$ index $P[j]$. By construction of indexP[.], $i=\operatorname{layer}(I)$, or $i=+\infty$ and $I \in E_{\text {last }}$. Similarly, $i^{\prime}=\operatorname{layer}\left(I^{\prime}\right)$, or $i^{\prime}=+\infty$ and $I^{\prime} \in E_{\text {last }}$. As a result, $I$ dominates $I^{\prime}$ with $S=O p t$ (resp. $S=P e s$ ) if and only if $i<i^{\prime}$ (resp. $i>i^{\prime}$ ).

Complexity. Checking if an outcome satisfies a preference is constant time. Thus, building violated $(P, I)$ and $\operatorname{violated}\left(P, I^{\prime}\right)$ is linear in $|P|$. Computing the maximum values $i$ and $i^{\prime}$ is done by a traversal of the structure index $P[\cdot]$, which is linear in $|P|$ as well.

\section{CONCLUSION}

We have studied the theoretical complexity of the main problems in optimistic/pessimistic preference logic: undominated, consistency, and dominance. We have shown that these problems are NP-hard in general, though they become polynomial for some specific semantics. We also show that, interestingly, the dominance problem, which is the only problem beyond NP and which in addition contains an online part, can be compiled to polynomial time. This opens the door to the use of CLP-nets in applications where we need to rank sets of outcomes on the fly.

\section{REFERENCES}

[1] C. Gonzales and P. Perny, "GAI networks for utility elicitation," in $K R$, D. Dubois, C. A. Welty, and M.-A. Williams, Eds. AAAI Press, 2004, pp. 224-234.

[2] C. Boutilier, R. I. Brafman, H. H. Hoos, and D. Poole, "Reasoning with conditional ceteris paribus preference statements," in Uncertainty in Artificial IntelligenceI, K. B. Laskey and H. Prade, Eds. Morgan Kaufmann, 1999, pp. 71-80. 
[3] J. Goldsmith, J. Lang, M. Truszczynski, and N. Wilson, "The computational complexity of dominance and consistency in cp-nets," J. Artif. Intell. Res. (JAIR), vol. 33, pp. 403-432, 2008. [Online]. Available: http://dx.doi.org/10.1613/jair.2627

[4] S. D. Prestwich, F. Rossi, K. B. Venable, and T. Walsh, "Constraint-based preferential optimization," in Proceedings, The Twentieth National Conference on Artificial Intelligence and the Seventeenth Innovative Applications of Artificial Intelligence Conference, July 9-13, 2005, Pittsburgh, Pennsylvania, USA, M. M. Veloso and S. Kambhampati, Eds. AAAI Press / The MIT Press, 2005, pp. 461-466. [Online]. Available: http://www.aaai.org/Library/AAAI/2005/aaai05-073.php

[5] C. Boutilier, R. I. Brafman, C. Domshlak, H. H. Hoos, and D. Poole, "CP-nets: A tool for representing and reasoning with conditional ceteris paribus preference statements," Journal of Artificial Intelligence Research, vol. 21, pp. 135-191, 2004.

[6] J. Pearl, "System Z: A natural ordering of defaults with tractable applications to nonmonotonic reasoning," in Proceedings of the 3rd Conference on Theoretical Aspects of Reasoning about Knowledge, Pacific Grove, CA, March 1990, R. Parikh, Ed. Morgan Kaufmann, 1990, pp. 121-135.

[7] S. Benferhat, D. Dubois, S. Kaci, and H. Prade, "Bipolar representation and fusion of preferences on the possibilistic logic framework," in Proceedings of the Eights International Conference on Principles and Knowledge Representation and Reasoning (KR-02), Toulouse, France, April 2225, 2002, D. Fensel, F. Giunchiglia, D. L. McGuinness, and M. Williams, Eds. Morgan Kaufmann, 2002, pp. 421-448.

[8] C. Boutilier, "Toward a logic for qualitative decision theory," in Proceedings of the 4th International Conference on Principles of Knowledge Representation and Reasoning (KR'94). Bonn, Germany, May 24-27, 1994., J. Doyle, E. Sandewall, and P. Torasso, Eds. Morgan Kaufmann, 1994, pp. 75-86.

[9] S. Kaci and L. van der Torre, "Algorithms for a nonmonotonic logic of preferences," in Symbolic and Quantitative Approaches to Reasoning with Uncertainty, 8th European Conference, ECSQARU 2005, Barcelona, Spain, July 6-8, 2005, Proceedings, ser. Lecture Notes in Computer Science, L. Godo, Ed., vol. 3571. Springer, 2005, pp. 281-292.

[10] S. Kaci and L. van der Torre, "Reasoning with various kinds of preferences: logic, non-monotonicity, and algorithms," Annals of Operation Research, vol. 163, no. 1, pp. 89-114, 2008.

[11] M. Bienvenu, J. Lang, and N. Wilson, "From preference logics to preference languages, and back," in Principles of Knowledge Representation and Reasoning: Proceedings of the Twelfth International Conference, KR 2010, Toronto, Ontario, Canada, May 9-13, 2010, F. Lin, U. Sattler, and M. Truszczynski, Eds. AAAI Press, 2010. [Online]. Available: http://aaai.org/ocs/index.php/KR/KR2010/paper/view/1360

[12] S. Benferhat and K. Souhila, "A possibilistic logic handling of strong preferences," in IFSA World Congress and 20th NAFIPS International Conference, 2001. Joint 9th, vol. 2, July 2001, pp. 962-967 vol.2.
[13] S. Benferhat, D. Dubois, and H. Prade, "Representing default rules in possibilistic logic," in Proceedings of the 3rd International Conference on Principles of Knowledge Representation and Reasoning (KR'92). Cambridge, MA, October 25-29, 1992., B. Nebel, C. Rich, and W. R. Swartout, Eds. Morgan Kaufmann, 1992, pp. 673-684.

[14] M. Cadoli and F. M. Donini, "A survey on knowledge compilation," AI Commun., vol. 10, no. 3-4, pp. 137-150, 1997. [Online]. Available: http://content.iospress.com/articles/aicommunications/aic133

[15] P. Liberatore, "Compilation of intractable problems and its application to artificial intelligence," Ph.D. dissertation, Dipartimento di Informatica e Sistemistica, Universitá di Roma "La Sapienza", 1998. 